\title{
Algunas consideraciones sobre el panorama actual de la lingüística clínica*
}

\author{
Some considerations about the current panorama of \\ Clinical Linguistics
}

\section{Josaphat Enrique Guillén Escamilla**}

\begin{abstract}
RESUMEN
A partir de una revisión bibliográfica, el objetivo de este trabajo es presentar un panorama general acerca del desarrollo actual de la lingüística clínica. Se inicia con la descripción teórica propuesta por Crystal para después discutir el problema de inteligibilidad que se presenta entre las diferentes disciplinas que intervienen en el estudio de las alteraciones del lenguaje. Posteriormente, se destaca el carácter teórico funcional que se ha estado adoptando dentro del contexto clínico. Luego, se describe el estado actual de la lingüística clínica en el ámbito hispánico y se enfatiza la carencia de este tipo de estudios en Latinoamérica. Finalmente, se concluye que es necesario fomentar el desarrollo de este tipo de investigaciones en la región, pues la gran mayoría de los resultados y datos con los que contamos provienen exclusivamente de la variante del español peninsular.
\end{abstract}

\begin{abstract}
This article aims to present an overview of the current development of Clinical Linguistics from a bibliographic review. We start with a description of Crystal's framework for, later, discuss the problem of intelligibility, which is present between the different disciplines interested in studying language disturbances. Then, emphasizing the functional theoretical character, which has been adopting within the clinical context. It is also described the current state of Clinical Linguistics in Hispanic ambit, highlight-
\end{abstract}

Palabras clave: alteraciones del lenguaje, lingüística aplicada, pragmática clínica.

Keywords:

Applied

Linguistics,

Clinical

Pragmatics,

Language

disturbance.

* Este trabajo se inscribe en el proyecto "Desarrollo de la lingüística clínica en México", realizado en la Escuela Nacional de Antropología e Historia, en la Licenciatura de Lingüística.

** Mexicano. Doctor en Lingüística. Académico Escuela Nacional de Antropología e Historia, Ciudad de México, México. josaphat_guillen@inah.gob.mx. 
Algunas consideraciones sobre el panorama actual de la lingüística clínica | Guillén Escamilla

ing the shortage of this kind of research in Latin America. Finally, we conclude that it is necessary to develop this kind of research in this region because the most results and data we have about language disturbances in Spanish belong to peninsular Spanish. 


\section{Introducción}

La lingüística clínica (en adelante LC), como una subárea de la lingüística aplicada, comenzó a ganar notoriedad durante la década de 1970 (Perkins, 2011) y, desde entonces, se ha tratado de reflejar su estatus "as a subdiscipline of linguistics rather than of speech and language pathology" (Ball, Perkins, Müller \& Howard, 2008, p. xxiv). En este sentido, aunque autores como Grunwell (1988) señalan que la lingüística llegó tarde al estudio de las alteraciones del lenguaje, hay que recordar que Jakobson (1941; 1955; 1966), desde la década de los 40, se había interesado en el análisis lingüístico de las afasias. Más todavía, para 1963, Trim ya había señalado cuáles eran las principales tareas de una aproximación lingüística a los desórdenes del lenguaje, a saber: observación precisa del habla de los pacientes; el análisis del sistema lingüístico deficitario que está siendo empleado por los pacientes; y la descripción de cómo se encuentra afectado el lenguaje en las situaciones comunicativas en las que participa el paciente.

Ahora bien, a pesar de su corta historia, se ha apuntado que la LC ha atravesado por tres etapas principales: prelingüística, gramatical y pragmática (Barroso y Nieto, 1996; Duchan, 2011; Fernández, 1998; Grunwell, 1988). La primera, que llega hasta antes de 1970, es considerada prelingüística porque el interés estaba centrado únicamente en las alteraciones de la pronunciación, sin prestar atención a los sustratos lingüísticos de estas fallas. La segunda etapa, que se extendió a lo largo de la década de los 70, se considera gramatical porque se presentó una preocupación por el estudio de los déficits lingüísticos, bajo el cobijo de la línea teórica trazada por Chomsky. En este periodo, Crystal y sus colegas desarrollaron técnicas y estudios para el análisis de la fonología, la morfología, la sintaxis, la semántica y la prosodia (Crystal, 1979; 1982; Crystal, Fletcher \& Garman, 1976). En estas dos primeras etapas abundaron los estudios acerca del hemisferio izquierdo y los niveles más básicos del lenguaje.

Por último, a finales de los 70 y principios de los 80 , se presentó la "revolución pragmática" (Duchan, 2011) o el "enfoque funcional" (Grunwell, 1988) e irrumpe un interés creciente por los aspectos pragmáticos y discursivos, por lo que las funciones de uso y la comunicación se volvieron prioritarias (Fernández, 1998). Posteriormente, a finales de los 90 y principios del 2000, se empieza a reconocer el papel del hemisferio dere- 
cho en la organización del discurso, en la interpretación de implicaturas conversacionales, de actos de habla indirectos y de lenguaje figurado, entre otros fenómenos pragmáticos (Joanette \& Ansaldo, 1999; Myers, 2001; Paradis, 1998). En consecuencia, se traza la diferencia entre desórdenes lingüísticos, asociados con el hemisferio izquierdo, y desórdenes pragmáticos, asociados con el hemisferio derecho.

No obstante, a pesar de esta división, actualmente se ha señalado que:

The scope of clinical linguistics is broad, to say the least. No level of language organisation, from phonetics to discourse, is immune to impairment, with problems manifested in both the production and comprehension of spoken, written and signed language across the human lifespan (Perkins \& Howard, 2011, p. 111).

Por lo anterior no tiene que entenderse que los estudios en LC deben excluir una u otra aproximación (gramatical o pragmática); más bien, hoy en día, se aboga por incluir en los análisis todos los componentes del lenguaje, desde el fonético y fonológico hasta el pragmático y el discursivo.

Así pues, en este contexto, el objetivo de nuestro trabajo es destacar algunos puntos fundamentales de la LC, en específico nos interesa: describir la teoría estándar propuesta por Crystal (sección 2); plantear los problemas que supone una perspectiva interdisciplinar (sección 3); presentar las características de una aproximación funcional en LC (sección 4); y mostrar, aunque sea de forma general, el panorama actual de esta disciplina dentro del ámbito hispánico (última sección).

\section{Hacia el establecimiento de una nueva rama de la lingüística aplicada}

Como se ha descrito en otros estudios, la lingüística aplicada está relacionada con la investigación empírica y teórica de los problemas sociales del mundo real donde el lenguaje desempeña un papel central (Brumfit, 1995; Davies \& Elder, 2004; Grabe, 2002). Entre estos problemas, el de las alteraciones del lenguaje siempre ha llamado la atención de los investigadores, quienes han intentado responder algunas de las siguientes preguntas: ¿cómo mejorar el diagnóstico y la evaluación de las alteraciones del lenguaje?, ¿cómo se puede contribuir a ofrecer mejores tratamientos de rehabilitación a personas con este tipo 
de déficits?, y ¿qué materiales se pueden desarrollar para mejorar la intervención clínica, tanto en la evaluación como en el diagnóstico y en la rehabilitación? De tal forma, resulta claro que "los trastornos del lenguaje no se enfrentan como un fenómeno abstracto, sino concretamente ligados a personas que lo sufren" (Pavez, 1998, p. 955).

En este escenario, a principios de los años 70 se empezó a delinear una nueva rama de la lingüística aplicada, interesada en investigar los hechos lingüísticos que ocurren dentro del ámbito hospitalario: la LC, cuya labor podría privilegiar uno de dos caminos: la aplicación de la teoría lingüística a la evaluación e intervención del lenguaje deficitario en contextos clínicos, o bien, teorizar acerca de cómo trabaja el lenguaje, el habla y la cognición en condiciones naturales, a partir del estudio de la patología (Müller \& Ball, 2013, p. 4).

Así pues, en esos años, David Crystal comenzó a pugnar por una "nueva fase" en la relación lingüística-terapia del lenguaje, "in which the relevance of linguistics will come to be viewed less idealistically and more practically than in the past" (1972, p. 4), ya que la lingüística podría ayudar a comprender los desórdenes del lenguaje en tres sentidos fundamentales: empíricamente, a través del planteamiento de nuevas hipótesis sobre la adquisición y la pérdida del lenguaje; procedimentalmente, proporcionando nuevas técnicas y métodos de análisis; y teóricamente, planteando nuevas explicaciones para la forma en que el lenguaje puede estar alterado en la patología (Crystal, 1972). A partir de estas consideraciones, se trazaron las tareas que la LC debía realizar: en primera instancia describir el desarrollo típico del lenguaje; luego describir el lenguaje en adultos sin patología; describir las características lingüísticas de los desórdenes del lenguaje; aportar técnicas descriptivas para ser empleadas en casos específicos; diseñar escalas evaluativas; proponer principios explicativos para la adquisición y la pérdida del lenguaje; y por último, redactar libros de texto introductorios a la lingüística, dirigidos a los profesionales de otras áreas.

Todos estos primeros planteamientos se vieron sintetizados a inicios de los años 80, cuando Crystal (1981) definió y presentó formalmente a la LC:

Clinical linguistics is the application of the theories, methods and findings of linguistics (including phonetics) to the study of those 
clinical situations where disorders of language are diagnosed and treated. 'Language', in this context, subsumes all four modes of interaction: speech, listening, reading and writing. 'Clinical', in this context, subsumes all those situations where there is a remedial intention involved: the initial focus is on medical settings, but educational, psychological and social settings where remedial language work is carried on are by no means excluded (p. 1).

Años más tarde, añadió que una de las finalidades de la LC sería estudiar la discapacidad (disability) del lenguaje en todas sus formas, refiriéndose con esto a aquellos casos que, por su complejidad, requieren un tratamiento o un método de enseñanza particular y, sobre todo, exigen un tipo de investigación especializado (Crystal, 2001; 2019). Así pues, la LC surgió con la intención de coadyuvar con el diagnóstico, la evaluación y el tratamiento de las alteraciones del lenguaje en contextos clínicos y, desde sus comienzos y hasta ahora, el aspecto aplicado sigue siendo su característica dominante (Crystal, 2013, p. 236). En resumen, "the chief aim of clinical linguistics is to provide the clinician with increasing levels of insight and confidence in arriving at linguistic decisions" (Crystal, 2001, p. 679).

Más recientemente, se ha postulado que el objetivo final de la LC es caracterizar las alteraciones del lenguaje de la misma forma en que la teoría lingüística general busca establecer universales lingüísticos (Crystal, 2019, p. 351), para lo cual es necesario realizar estudios comparativos con otras lenguas distintas del inglés. Así, se requiere que los investigadores realicen, en diferentes lenguas, descripciones precisas de la producción de los pacientes y que registren y clasifiquen esta información para contribuir, así, con el estudio comparativo.

Finalmente, como una disciplina aplicada, la LC tiene cinco metas principales: clarificación, descripción, diagnóstico, evaluación e intervención (Crystal, 2019, p. 352). El primer aspecto está relacionado con la necesidad de clarificar los conceptos y la terminología de las distintas disciplinas que interactúan en la LC. Esto es, debe existir una inteligibilidad entre los distintos profesionales, tanto teórica como metodológicamente, para establecer un punto de partida común que incorpore explicaciones precisas y criterios explícitos. Por su parte, la descripción supone establecer pautas que permitan analizar y detallar la conducta lingüística de los pacientes, de los familiares y de los 
clínicos que interactúan con ellos. Como muestra de esta necesidad, por ejemplo, hasta hace poco no eran comunes los estudios de caso dedicados a la descripción cualitativa de la producción lingüística de los pacientes; más bien, la tendencia había sido privilegiar los análisis cuantitativos con muestras muy amplias de pacientes, pues se consideran "más válidos" por ser generalizables (Felson, 2014).

En cuanto al diagnóstico, la LC puede proveer una clasificación clara de los déficits lingüísticos que presenta el paciente, esto como parte del proceso del diagnóstico diferencial, ya que se ha demostrado que un modelo puramente médico de investigación no es suficiente para explicar las alteraciones del lenguaje ${ }^{1}$. Así,

A linguistically informed classification -in which a language disability is characterized explicitly with reference to its use of phonetic, phonological, grammatical, semantic, pragmatic, and other variables - can provide an alternative diagnosis model and one that is more able to provide insights about intervention in cases where there is no clear evidence of any medical condition (Crystal, 2019, p. 353).

Respecto del cuarto punto, la LC contribuye a elaborar una evaluación más sofisticada y precisa de los déficits lingüísticos; así, mientras el diagnóstico revela qué es lo que está mal en la producción o comprensión del paciente, la evaluación determina qué tan serio es eso que está alterado en el lenguaje del paciente. Finalmente, la LC puede auxiliar a los clínicos a realizar una mejor intervención a partir del análisis del habla del paciente, pero también del de la persona que lo cuida, del tipo de materiales empleados y de las situaciones en las que tiene lugar la interacción comunicativa.

Hasta aquí, hemos presentado el desarrollo de lo que se podría llamar "teoría estándar" de la LC, cuyo principal impulsor ha sido David Crystal. No obstante, desde finales de los años 80, otros inves-

1 No obstante, algunos investigadores han advertido que: "Crystal's early distinction between a 'linguistic model' (the characterization of communication disorders in exclusively linguistic terms) and a 'medical model' (the underlying 'medical' causes of the disorders), while still having considerable heuristic value, nevertheless presents a somewhat fragmentary and dissociative view" (Perkins, 2011, p. 926), por lo que actualmente no se aconseja establecer esta división entre diagnóstico médico y diagnóstico lingüístico; más bien, se pugna por un diagnóstico que integre ambas visiones. 
tigadores han comenzado a reorientar algunos aspectos teóricos y han pugnado por un estudio bidireccional, esto es, que siga estando presente la aplicación de la teoría y las herramientas metodológicas de la lingüística, pero que, además, se consideren los datos clínicos y su probable contribución a la teoría lingüística general (Ball \& Kent, 1987; Howard, 2002; Perkins \& Howard, 1995). En este sentido, debemos advertir que los resultados clínicos servirían, en primera instancia, para comprobar las hipótesis postuladas desde la psicolingüística; posteriormente, serían integrados a la agenda de la neurolingüística y, finalmente, de ser el caso, se presentaría la incorporación a la lingüística general. Así pues, los datos clínicos recorren su propio camino, a lo largo de distintas ramas, antes de poder ser considerados por la lingüística general.

\section{La inteligibilidad y los problemas de la interdisciplinariedad}

Recientemente se ha señalado la necesidad de aclarar el estatus epistemológico de la LC, a partir de la distinción entre multidisciplinariedad, interdisciplinariedad e intradisciplinariedad (Jiménez, 2019). En el primer caso, son varias disciplinas, además de la lingüística, las que se ocupan del estudio de las alteraciones del lenguaje, pero cada una lo hace desde su perspectiva y sin interacción entre ellas. En contraste, en la interdisciplinariedad se presenta una relación con otras áreas de conocimiento con las que se comparte el objeto de estudio; en el caso de la LC, la interacción se da entre la lingüística, la neurología, la psicología, la neuropsicología, la foniatría y la logopedia. En cuanto a la intradisciplinariedad, es desde la lingüística donde se especifica la relación entre la LC y otras ramas; en particular, la psicolingüística, la neurolingüística, la afasiología lingüística y la biolingüística. A partir de estas distinciones, se propone una transversalidad que funge como un organizador metodológico de todas esas otras áreas de conocimiento y que, junto con la interdisciplinariedad, permite el análisis integral de las alteraciones del lenguaje (Jiménez, 2019).

De tal forma, como parte de las neurociencias cognitivas, la LC exige la construcción de un marco teórico interdisciplinar, pues su objeto de estudio - las alteraciones del lenguaje - trasciende al ámbito de una sola área de investigación. Por ejemplo, en el caso de la neuro- 
lingüística, Harry Whitaker (1971) aseguraba que el progreso de esta disciplina dependía de que se lograra establecer una sinergia exitosa entre lingüística y neurología, por lo que, a partir de los años 70 se empezaron a llevar a cabo las primeras colaboraciones interdisciplinarias entre psicólogos, lingüistas y neurocientíficos (Kemmerer, 2015, p. 3). Así, en el estudio de las alteraciones del lenguaje, convergen varias ciencias interesadas en contribuir a la explicación integral de los déficits del lenguaje dentro de cuadros patológicos. En consecuencia, se espera que todo investigador involucrado en este tipo de estudios tenga "una formación profesional lo más interdisciplinar posible" (Marrero y Martín, 2005, p. 209).

En este sentido, Garayzábal (2004) apunta que la LC tiene una existencia propia y autónoma dentro de la lingüística, pero no puede deslindarse de otras áreas afines, por lo que, desde la lingüística se ha rechazado que pueda existir una LC independiente que, de forma aislada, sea capaz de diseñar estrategias de evaluación e intervención. De tal forma, "Su abordaje requiere un enfoque distinto al utilizado usualmente en lingüística y exige además conocer otros aspectos que coexisten con los diversos trastornos (aspectos psicológicos, neurológicos, fisiológicos, etc.)" (Pavez, 1998, p. 954). En resumen, la LC debe ser concebida como un área que está vinculada con un amplio rango de disciplinas que van más allá de la lingüística (Perkins, 2011).

No obstante, esto que se defiende en la teoría, no siempre se cristaliza en la práctica, ya que muchas veces la interdisciplinariedad se puede confundir con intrusismo (Gallardo, 2002; Garayzábal, 2004) y, en realidad, lo que sucede es que cada investigador sigue persiguiendo, cada uno por su lado, sus propios fines e intereses. Además, otro factor que debe considerarse es que tradicionalmente el lingüista no ha sido invitado o no ha estado interesado en asistir a estos cuerpos interdisciplinares y se ha obviado la aportación de la lingüística, como si la ciencia del lenguaje no tuviera nada que decir acerca de las alteraciones del lenguaje. Un ejemplo de esto es lo que sucedía en las investigaciones en torno a la afasia, donde: "the classical studies of aphasia were conducted by neurologists and neuropsychologists who had no specialized linguistic training [...] Similarly, linguists formulated their theories of human language independently of any serious considerations of language loss in aphasia" (Ingram, 2007, p. 4). Así, ha sido más 
sencillo hablar de interdisciplinariedad en el discurso que en los hechos y esto ha repercutido en la agenda de la LC.

Como ya hemos establecido, el carácter interdisciplinar de la LC obliga al intercambio con otros especialistas: neuropsicólogos, logopedas, médicos, fonoaudiólogos, terapeutas, profesores, etc., pero para que esta relación sea realmente fructífera es necesario que "el lingüista [comparta] la terminología básica de los otros profesionales y, a su vez, usar un lenguaje técnico y una conceptualización accesibles para los demás" (Pavez, 1998, p. 955). Justo, aquí sostenemos que el problema de la interdisciplinariedad es, al menos en parte, uno de ininteligibilidad, ya que los investigadores - provenientes de distintas áreas- no comparten una lingua franca que les permita entenderse entre ellos, y esto ha supuesto un gran obstáculo al momento de intentar alcanzar sus objetivos. En palabras de Pulvermüller (2002):

Linguists may have difficulty understanding the language of neuroscientists, and, conversely, neuroscientists may have difficulty understanding linguistic terminology. After all, the distance between linguistics and neuroscience is not smaller than that between physics and chemistry. Given that such mutual comprehension difficulty is relevant, the important problem may be a problem of translation (p. 271).

En este mismo sentido, Marrero y Martín (2005) han señalado que una mala comunicación interdisciplinaria ha provocado que "la psicología del lenguaje y, en concreto, la ciencia que se ocupa de las patologías del lenguaje y la comunicación se hayan visto invadidas por términos mal traducidos, que han ido desplazando a los términos correctos" (p. 212). Así pues, sigue siendo una tarea pendiente la construcción de un marco teórico común, transversal, que ayude a explicitar la terminología de las distintas áreas que intervienen en el estudio de las alteraciones del lenguaje (Serra, 2013).

Otro problema relacionado con esto es el que Quilis y Hernández (1990) señalaron ya hace varios años atrás en cuanto a que el estudio de las alteraciones del lenguaje había congregado a maestros, psicólogos, psiquiatras, entre otros especialistas, pero la ausencia de lingüistas era muy evidente "porque cuando se repasaba la bibliografía, el aspecto lingüístico del tema es bien pobre y vago" (p. 7). Más aún, se 
ha destacado que la LC adolece de una profesionalización, ya que su desarrollo se ha circunscrito al ámbito clínico, soslayando el papel de la lingüística. Así, parte del problema es que la "discrepancia y heterogeneidad en las bases teóricas y metodológicas es lo que, salvo excepciones, nos ofrece la bibliografía general sobre patología del lenguaje" (Gallardo y Valles, 2008, p. 45).

Por otra parte, las pruebas empleadas para obtener perfiles diagnósticos también reflejan esta carencia, pues acusan una falta de rigor teórico dado que ningún lingüista intervino en su elaboración; así, es común que se tomen como errores aquellos aspectos que los pacientes no han sabido o podido responder y "que por edad, nacionalidad, sexo, etc. no tenían por qué ser correctamente respondidos" (Garayzábal, 2004, p. 173). Por todo esto, se ha abogado para que los lingüistas intervengan en el diseño de este tipo de materiales, empleando las teorías y los métodos de la lingüística y atendiendo a la naturaleza específica del problema al que se enfrentan (Pavez, 1998).

Ahora bien, todo lo que hemos dicho hasta ahora acerca de la exclusión de la lingüística del ámbito clínico puede ser atribuido, en el sentido contrario, a los lingüistas, es decir, son pocos los que se han interesado por ocuparse ellos mismos de aprender las metodologías y los conceptos de la neuropsicología, la psicología, la medicina, la fisiología, etc. Son pocos los que han asistido a la clínica y analizado directamente la producción y la comprensión de los pacientes, intentando cooperar con los clínicos para delinear, por ejemplo, el rumbo que debería tomar la rehabilitación. Evidentemente, esto también ha detenido el avance de la investigación y ha puesto en evidencia la falta de apertura de algunos lingüistas:

Linguistics is the study of language. Language is a system of brain circuits [...] If linguistics is the study of language and language is in one sense a system of brain circuits, one would expect linguists to be open to the study of brain circuits. However, the dominating view in linguistics appears to be that language theories must be formulated in an abstract manner, not in terms of neuron circuits (Pulvermüller, 2002, p. 270).

A esta crítica se han unido otros autores, como Damico y Simmons-Mackie (2003), quienes han señalado que el campo clínico está 
consciente de la necesidad de incluir a otras áreas, pero lo que ha prevalecido, en cambio, es una división entre la investigación teórica (lingüística) y la práctica clínica, esto a pesar de que se ha reconocido que los factores lingüísticos son indispensables y están íntimamente relacionados con el diagnóstico neuropsicológico (Lawless, Ries \& Llorente, 2008). En breve, 'clinical work' is not separable from the scientific work done by linguists; instead, it should be considered as a component part of General Linguistics" (Fava, 2002, p. x).

Otro dilema más está asociado con lo heterogéneo de la teoría lingüística, lo que per se no debería juzgarse como problemático sino como enriquecedor, pero se ha llegado a considerar que lo único que tienen en común los lingüistas es su interés por lo datos clínicos (Müller \& Ball, 2013) y están muy lejos de proponer una base teórica común que sirva como punto de partida para las investigaciones clínicas. No obstante, esto mismo se podría decir de cualquiera de las otras ciencias; por ejemplo, en el ámbito neuropsicológico hay tradiciones que privilegian los estudios cuantitativos y los aspectos fisiológicos, mientras que otras prefieren los estudios de caso y la descripción cualitativa de los déficits lingüísticos y su relación con los procesos fisiológicos dañados. En cualquier caso, lo que se requiere es una congruencia teórica entre las aproximaciones empleadas para evitar, por ejemplo, un análisis pragmático desde una perspectiva chomskiana.

Finalmente, queremos concluir este apartado matizando las palabras de Garayzábal (2004) cuando mencionaba que, en los albores de este milenio, los problemas de la interdisciplinariedad redundaban

en la gran dificultad para encontrar foros comunes de interés así como lugares de publicación donde la postura y el hacer de la lingüística clínica se tome en serio y en sus justos términos de aportación de información relevante al mundo científico dedicado a las alteraciones del lenguaje (p. 177).

Si bien es cierto que los foros no son abundantes como ocurre en otras áreas de la lingüística, cada vez hay más cabida para la LC en revistas y congresos de lingüística, logopedia, fonoaudiología, neuropsicología, etc. En el caso de las revistas, desde 1987 se publica Clinical Linguistics \& Phonetics; y, en el ámbito hispánico, en España se edita la Revista de Investigación en Logopedia y la Revista de Logopedia, Fonia- 
tría y Audiología. En Chile se publica la Revista Chilena de Fonoaudiología; en Argentina cuentan con la Revista Fonoaudiológica Argentina; en Colombia tienen la Revista Areté y, en México, hasta 2017, se editó la Revista Mexicana de Comunicación, Audiología, Otoneurología y Foniatría. Por otra parte, desde 2006, en España se celebra cada tres años el Congreso Internacional de Lingüística Clínica, evento que se ha convertido en un testimonio de cómo ha ido evolucionado tanto la concepción de LC, como el tipo de investigaciones que se realiza dentro del ámbito hispánico. Esto gracias a que, en cada edición, una selección de los trabajos más destacados se publica en formato de libro. En resumen, la tarea ahora es abrir más espacios para la discusión y la difusión del quehacer de la LC, sobre todo en América Latina.

\section{Un enfoque funcional y comunicativo en la LC}

En las últimas dos décadas ha ido en aumento el rechazo a las explicaciones lingüísticas tradicionales que privilegian el análisis de la competencia lingüística sobre el de la actuación, ya que se han mostrado poco útiles para evaluar lo que pasa realmente en el ámbito clínico o, en el mejor de los casos, solo guían a conclusiones parciales (Gallardo y Hernández, 2013). Por tal motivo, resulta natural que la inclusión del análisis del componente pragmático sea reciente y todavía escaso, a pesar de ser un ámbito de investigación bastante fértil (Crystal, 2013; Powell \& Ball, 2010), sobre todo si consideramos que un daño pragmático se presenta, a la vez, como un fenómeno de índole social y cognitivo (Perkins, 2014).

En este contexto, desde hace más de diez años, se han postulado dos términos, pragmática clínica y neuropragmática, para referirse al estudio de los mecanismos cerebrales cuyo daño guía a una conducta pragmática atípica, con lo que se intenta entender cómo el cerebro comprende y produce la conducta pragmática lingüística (Perkins, 2014; Stemmer, 2008). Con todo, la pragmática clínica ha sido más reconocida como parte de la LC que la neuropragmática, a pesar de que ambas comparten los mismos intereses de investigación. A este respecto, Perkins (2014) apunta que:

clinical pragmatics is a subdiscipline of clinical linguistics, which in turn is a branch of applied linguistics concerned with the ways in which communication may be impaired [...] The term 'clinical 
pragmatics' will be used here to refer to the study of pragmatic ability in individuals with communication disorders. It covers the description and classification of pragmatic impairments, their elucidation in terms of various pragmatic, linguistic, psychological and neurological theories, and their assessment and treatment (p. 66).

Así pues, a partir de la inclusión de la pragmática, en la LC se estableció una diferencia entre lenguaje (competencia lingüística) y comunicación (competencia comunicativa), el primero asociado con el hemisferio izquierdo y la segunda, con el hemisferio derecho ${ }^{2}$ (Joanette \& Ansaldo, 1999; Myers, 2001; Paradis, 1998; Stemmer, 2008), como si un déficit lingüístico no pudiera traer consigo una alteración pragmática o como si ambas competencias se excluyeran entre sí. De tal forma, se han postulado términos como disiponoia (Paradis, 1998), afasia pragmática (Joanette \& Ansaldo, 1999) y apragmatismo (Myers, 2001) para referirse a aquellas alteraciones pragmáticas provocadas por un daño en el hemisferio derecho, aunque el propio Paradis (1998) considera que un daño en el hemisferio izquierdo también puede provocar un daño pragmático, pero de segundo orden, es decir, se presenta como resultado de un déficit lingüístico o sensomotor y no como uno pragmático per se. En este sentido, Perkins (2014) subraya que:

'pragmatic impairment' is most commonly used with reference to conditions such as right hemisphere brain damage and autism, which affect socio-cognitive more than linguistic functioning, pathologies of speech and language such as aphasia and developmental language disorder may also be regarded as having a pragmatic dimension to the extent that they restrict the choices available to an individual to produce and/or comprehend utterances in accordance with the requirements of the communicative situation (p. 67).

2 A este respecto, Gallardo (2002) distingue entre competencia gramatical y competencia pragmática. La primera incluye los niveles de la lengua, desde la fonética hasta la semántica, que pueden verse alterados ante un cuadro patológico. Por su parte, la segunda incluye las habilidades comunicativas que pueden alterarse en la patología. La utilidad metodológica de esta precisión se ha visto reflejada en el Protocolo rápido de evaluación pragmática-revisado (Fernández-Urquiza, Díaz, Moreno, López y Simón, 2015). 
En resumen, se tiene que evitar caer en el reduccionismo de asociar una alteración pragmática solo con el hemisferio derecho y una alteración lingüística solo con el hemisferio izquierdo, ya que ambos hemisferios intervienen durante el procesamiento de factores pragmáticos, desde los relacionados con la coherencia y la cohesión, hasta los vinculados con la interpretación de la prosodia o el lenguaje figurado.

Por otro lado, hay que enfatizar que, al adoptar un marco teórico pragmático, la LC asume los siguientes planteamientos: el uso del lenguaje es funcional; su función es crear significados; y estos significados (funciones) están influidos por el contexto social y cultural en el que se desenvuelven (Müller, Mok \& Keegan, 2014). Además, supone incorporar el análisis del contexto y otros "conceptos como el conocimiento enciclopédico, conocimiento compartido, relevancia, inferencia, etc. [... por lo que] ya no se centra en esa 'habilidad lingüística formal', sino en la eficacia sociocomunicativa del sujeto" (Gallardo, 2002, p. 143).

Así pues, los desarrollos recientes en LC incluyen la identificación y el análisis de los mecanismos neurológicos y cognitivos que intervienen durante la producción y comprensión de los enunciados que componen un discurso, ya que se busca determinar cuáles son los sustratos neurofisiológicos de la coherencia y la cohesión, por ejemplo (Perfetti \& Frishkoff, 2008). Otros temas que han acaparado el interés de los investigadores son: la prosodia afectiva, el discurso narrativo y conversacional, el humor, los actos de habla indirectos, las metáforas, la ironía, la relevancia, la estructura de la información, los gestos, la postura, el conocimiento compartido y situacional, entre otros (Perkins, 2014; Stemmer, 2008). Además, recientemente se ha insistido en incluir variables sociales como el hábitat, el entorno familiar, el contexto escolar y la participación sociocomunicativa, entre otras, ya que "resultan claves para evaluar el desarrollo del lenguaje y para determinar en qué casos se halla retardado, desviado o limitado y no es resultado de variación particular por influjo de las variables" (Fernández, 2002, p. 17). Todos estos fenómenos son del interés de lo que Ball (2005) ha denominado sociolingüística clínica.

Otra parte importante de la investigación se ha centrado en el análisis de la interacción, en especial en la forma en que los pacientes emplean estrategias compensatorias para comunicarse de mejor manera, esto a pesar de que su identificación no sea tan simple (Perkins, 2014). 
Por ejemplo, Heeschen y Schegloff (citado en Wilkinson, 1986) han sugerido que

'telegraphic speech', a form of systematically simplified grammar observed in the talk of some types of 'no-fluent' aphasic speakers, may be understood no simply as a direct reflection of an underlying neurological or neuropsychological deficit but may be an adaptive turn-constructional method adopted by these speakers for interactional ends (p. 105).

En esta misma línea, Goodwin (2014) ha dicho que las limitaciones de los pacientes pueden ser superadas $\mathrm{o}$, al menos, mitigadas gracias a las estrategias compensatorias que surgen durante la interacción social cooperativa, cara a cara.

Como es de esperarse, la reciente inclusión del componente pragmático también se ha notado en las pruebas tradicionales empleadas para la evaluación del lenguaje, por lo que se ha criticado su aplicación indiscriminada ya que "no reflejan la magnitud real del problema" (Codesido, 2004, p. 6). Este tipo de pruebas están formuladas para medir la competencia gramatical, donde "it seems reasonable to conceptualize the speakers as an entity, frequently an isolated individual, capable of constructing syntactically complex sentences, with the human brain" (Goodwin, 2014, p. xi), sin considerar otros elementos de la interacción comunicativa.

En consecuencia, se ha insistido en que los datos deben ser obtenidos a partir de situaciones comunicativas reales y diversas para, posteriormente, realizar un análisis cualitativo que aporte información representativa de la competencia comunicativa de los pacientes. En palabras de Garayzábal (2004) "valorar el lenguaje patológico supone valorar el lenguaje desde una perspectiva natural, interactiva, y teniendo en cuenta al sujeto en sí, sin compararlo con nadie, ya que cada persona es única en su desarrollo" (p. 173). Más aún, conviene recordar - como lo señala Pietrosemoli (2007) — que la lengua se produce siempre en contexto, por lo que es sensible a él, esto explicaría el por qué

Muchas veces encontramos diferencias notables entre las habilidades que muestra un determinado hablante en situación que él reconoce como prueba evaluativa y en situación conversacional, 
incluso si ambas son realizadas por la misma persona, o el mismo equipo de personas (p. 315).

Finalmente, la contribución de la pragmática ha sido doble; por un lado, se ha empleado para el diseño y elaboración de pruebas y, por el otro, se han desarrollado pruebas específicas que evalúan diferentes aspectos del componente pragmático (Fernández, 1998).

Con todo, y a pesar del interés creciente en la pragmática, el número de investigaciones centradas en este tipo de fenómenos sigue siendo muy bajo en comparación con el resto de los tópicos en LC. Así, de acuerdo con Perkins (2011), hasta 2008 las investigaciones publicadas en Clinical Linguistics \& Phonetics estaban distribuidas de la siguiente manera: fonética (43\%), fonología (19\%), gramática (13\%), pragmática (7\%) y discurso (6\%). Una explicación para esto proviene desde la clínica y es que los desórdenes pragmáticos - en contraste con las alteraciones gramaticales - han sido mal diagnosticados o ni siquiera se han diagnosticado como problemas de orden lingüístico o psicológico: "Sophisticated syntactic accounts of disability emerged during the 1970s, and since then, there have been sporadic yet insightful applications of notions from semantics and pragmatics, once again, reflecting the (also sporadically insightful) state of the art in those subjects" (Crystal, 2019, p. 352).

También destaca que, en este mismo periodo, solo el $15 \%$ de los artículos estaba centrado en otra lengua distinta del inglés, esto a pesar de que Crystal (2013) ha dicho que

A multilingual perspective is a sine qua non of a clinic linguistics. The subject is clinical linguistics, not clinical English linguistics [...] The general point is that we need to broaden the descriptive base of our subject, with analyses relating to as many other language as possible (p. 238).

En resumen, actualmente existen dos carencias que subsanar: el bajo porcentaje de estudios centrados en la pragmática y el discurso, y el bajo número de investigaciones en lenguas distintas del inglés.

Finalmente, como se ha destacado a lo largo de este apartado, la LC ha ido adoptando un enfoque funcional para el estudio de las alteraciones del lenguaje, lo que ha supuesto la inclusión de factores pragmáticos y discursivos que hasta hace unos años habían sido ignorados. La 
influencia de esta inclusión se ha visto reflejada en tres aspectos principalmente: el análisis integral de los hechos lingüísticos que suceden en la clínica, tanto del lenguaje como pragmáticos; la consideración de la teoría pragmática durante la elaboración de las pruebas de evaluación; y la elaboración de pruebas que evalúan específicamente el componente pragmático. En este punto, queremos destacar dos protocolos que evalúan el componente pragmático y que se han originado en el ámbito hispánico: el Protocolo rápido de evaluación pragmática-revisado (Fernández-Urquiza et al., 2015) y MetAphAs, Protocolo de exploración de habilidades metalingüisticas naturales en la afasia (Rosell y Hernández, 2014). En el primer caso, los autores dividen la pragmática en tres: enunciativa, textual e interactiva; así, la prueba está diseñada para evaluar a cada subtipo por separado; mientras que el segundo evalúa las habilidades metalingüísticas naturales en personas con afasia, desde una perspectiva metacognitiva de la conducta verbal.

Por último, la propuesta es continuar con los estudios de los niveles más básicos del lenguaje, pero además, seguir incluyendo las consideraciones del análisis pragmático y discursivo como lo hacen los dos protocolos antes mencionados.

\section{La LC en el ámbito hispánico ${ }^{3}$}

España cuenta con una larga tradición en estudios de LC, donde destacan los grupos de investigación: Grupo Koiné (Universidad de Santiago de Compostela), que está interesado en la adquisición atípica del lenguaje; el Grupo de investigación en lingüística clínica (Universidad de Valencia), cuyo interés es muy variado y abarca desde el estudio de las afasias hasta los síndromes de Williams y Asperger y lesiones en el hemisferio derecho; el Gabinete de lingüística clínica (Universidad de Cádiz), donde se desarrollan investigaciones acerca de trastornos motores y trastornos de lenguaje y la enfermedad de Parkinson; el Proyecto de la mente bilingüe (Universidad del País Vasco), que investiga cómo se representa y procesa el lenguaje; y el Grupo login y el Proyecto syndrolig (Universidad de Oviedo), uno interesado en el lenguaje in-

3 No es nuestra intención hacer una descripción exhaustiva del estado actual de la LC en España; más bien intentamos presentar un panorama general que sirva para contextualizar al lector. Aquel que esté interesado en profundizar en esta descripción se recomienda consultar Fernández-Urquiza (2017). 
fantil y el otro en el estudio de los síndromes de Williams, de Down y de X-Frágil (Fernández-Urquiza, 2017).

Además, resaltan las figuras de Beatriz Gallardo-Paúls (Universidad de Valencia), Jordi Peña-Casanova, Faustino Diéguez (Universidad de Barcelona), Eliseo Díez Itza, Fernando Cuetos (Universidad de Oviedo), Elena Garayzábal (Universidad Autónoma de Madrid) - interesada en el estudio de los síndromes de Williams y el de Smith-Magenis-, Milagros Fernández (Universidad de Santiago de Compostela), Victoria Marrero (Universidad Nacional de Educación a Distancia), María Jesús Paredes Duarte (Universidad de Cádiz), entre otras. De acuerdo con Fernández-Urquiza (2017), la mayoría de estos investigadores parten de una base teórica funcional y permiten identificar tres áreas generales del quehacer de la LC: creación de perfiles lingüísticos de poblaciones clínicas, a partir de la elaboración de corpus; instrumentos de evaluación del componente pragmático; y la aplicación de las nuevas tecnologías en el ámbito de la LC.

En contraste, en Latinoamérica el panorama es bastante distinto. La LC ha tenido un desarrollo menos prolijo y esto se ve reflejado en el número reducido de investigadores e investigaciones, lo que puede ser atribuido en parte al

desconocimiento de su existencia, lo que redunda en la pobreza investigadora y educativa; pero lo que es aún más grave es el hecho de que a un nivel más particular, como es el de la propia Lingüística, se lleguen a desconocer o menospreciar estas mismas disciplinas emergentes (Garayzábal, 2004, p. 169).

Con todo, en este escenario podemos destacar, por un lado, a Lourdes Pietrosemoli (Universidad de Los Andes, Venezuela), quien ha enfatizado la importancia de incluir una perspectiva pragmática y discursiva al estudio de las afasias (1996; 2007; Pietrosemoli, Vera, González y Coutín, 2005). Y, por otro lado, a María Mercedes Pavez (Pontificia Universidad Católica de Chile), quien es pionera en el estudio lingüístico de las afasias en Chile (Pavez, 1978; 1980; 1982) y en destacar la importancia de la lingüística aplicada al estudio de los trastornos del lenguaje ( $\mathrm{Pa}-$ vez, 1998). Otros académicos que han desarrollado esta línea de investigación son Luis Martínez (Universidad de Talca, Chile), Beatriz Labos (Universidad de Buenos Aires, Argentina) y Ana Paula Mackay (Universi- 
dad de Santa Casa, Brasil) (Gallardo y Valles, 2008). En México, a inicios de los 70, se fundó el Grupo Mexicano de Estudios sobre Afasiología, coordinado por Raúl Ávila. Uno de sus aportes fue el Cuestionario para el estudio lingüistico de las afasias (Ávila, Berruecos y Durán, 1975), trabajo que significó la fundación de esta línea de investigación en aquel país. Actualmente, Donna Jackson, Alejandra Auza, Josaphat Guillén, entre otros investigadores, realizan estudios en esta área.

Por otro lado, también se ha dicho que parte del problema es la existencia de pocas escuelas de fonoaudiología o logopedia en Latinoamérica. Así, de acuerdo con Marrero (2008), Colombia es el país que tiene más programas (17), seguido por Argentina (16) y Chile (13), mientras que en otros países la presencia es más periférica: Bolivia (2), Perú (2), Ecuador (1). Incluso, recientemente se ha reconocido que solo Argentina, Chile, Brasil, Colombia, Venezuela, Cuba y Perú cuentan con una tradición en este tipo de estudios (Sandoval, 2018). Así pues, también se padece una falta de formación y profesionalización en LC, lo que ha estado deteniendo su avance. De tal forma, el camino que debe recorrer la LC en Latinoamérica es mayor, pues resulta necesaria la apertura de más escuelas y posgrados que aborden el estudio de las alteraciones del lenguaje, lo que a su vez podría provocar que el interés en este tipo de investigaciones aumente.

Finalmente, cabe destacar que resulta indispensable crear más foros especializados donde se puedan presentar y discutir las investigaciones surgidas dentro del ámbito latinoamericano. Al hacerlo, estaríamos contribuyendo a la construcción de una LC comparativa, aportando datos y resultados de las variedades del español de América, que son distintas entre sí y distintas del español peninsular, que es del que abundan los estudios. Así pues, todo esto podría contribuir, por ejemplo, a la elaboración de corpus para cada variante del español de América y facilitar así el estudio contrastivo, lo que en última instancia ayudaría a subsanar el carácter fragmentado de este tipo de investigaciones en América Latina.

\section{Consideraciones finales}

Hemos querido plantear un panorama, aunque fuera general, de la historia de la LC, de los problemas que enfrenta y de su quehacer actual dentro del ámbito hispánico. Se ha destacado su carácter interdiscipli- 
nar y los problemas de inteligibilidad que esto supone; además, se ha dicho que cualquier profesional interesado en el campo de la LC debe procurar una formación lo más integral posible. También se ha señalado la falta de desarrollo de la LC en Latinoamérica y lo importante que es resarcir esta carencia para poder describir los hechos lingüísticos que acontecen en las diferentes variedades del español de América. En este sentido, es una tarea pendiente la divulgación del quehacer de la LC, esto se debe en parte a que existen pocos cursos universitarios y de posgrado que oferten programas de estudios relacionados con ella y, además, a que esta área de investigación sigue siendo poco conocida en esta región del mundo (Cause y Bonne, 2017).

Así pues, en definitiva, el objetivo de la LC sigue siendo:

desarrollar una ciencia no solo teórica, sino también resolutiva, es decir, que aporte soluciones prácticas a la descripción de los desórdenes del lenguaje; que proporcione sistemas de transcripción eficaces y rápidos, así como métodos de evaluación fiables y ajustados a la realidad de la lengua; una ciencia que proporcione, asimismo, los medios que permitan diseñar terapias del lenguaje que no sean reduccionistas, es decir, que no se limiten a las bases orgánicas, cognitivas o puramente evolutivas del lenguaje, sino que también tengan en cuenta las características de la lengua que se trata de reeducar o estimular (Marrero y Martín, 2005, p. 210).

Finalmente, antes de concluir debemos apuntar la importancia de considerar al paciente como lo que es: un ser humano y no solo un objeto de estudio, ya que en algunos estudios "[they] are often depicted as objects, with the descriptions focusing on their test scores, communication disabilities, and speech or language progress, leaving out their voices or personal views" (Felson, 2014, p. 11). De tal forma, no debemos olvidar que estamos trabajando con personas y que, en última instancia, nuestra investigación busca contribuir a mejorar la calidad de vida de los pacientes con alguna alteración del lenguaje y la de sus familiares.

\section{Referencias bibliográficas}

Ávila, R., Berruecos, M., y Durán, J. (1975). Cuestionario para el estudio lingüístico de las afasias. México, D.F.: Instituto Mexicano de la Audición y el Lenguaje-El Colegio de México. 
Ball, M. (2005). Preface. En M. Ball (Ed.), Clinical Sociolinguistics (pp. xix-xx). India: Blackwell.

Ball, M. \& Kent, R. (1987). Editorial. Clinical linguistics \& phonetics, 1(1), 1-5. https://doi.org/10.1080/02699208708985000

Ball, M., Perkins, M., Müller, N., \& Howard, S. (2008). Introduction. En M. Ball, M. Perkins, N. Müller, \& S. Howard (Eds.), The handbook of clinical linguistics (pp. xxiii-xxxiv). Singapore: Blackwell.

Barroso, J. y Nieto, A. (1996). Asimetría cerebral: hemisferio derecho y lenguaje. Psicología Conductual, 4(3), 285-305.

Brumfit, C. (1995). Teacher professionalism and research. En G. Cook \& B. Seidlhofer (Eds.), Principle and practice in applied linguistics (pp. 27-41). Oxford: Oxford University Press.

Causse, M. y Bonne, A. (2017). Lingüística clínica: ¿una relación interdisciplinar poco conocida? Revista Habanera de Ciencias Médicas, 16(4), 635-643. Recuperado de http://www.revhabanera.sld.cu/index.php/rhab/article/view/2071/1850

Codesido, A. (2004). Revisión del cuestionario trastorno específico del lenguaje: métodos diagnósticos y evaluación. En M. Villayandre (Coord.), Actas del V Congreso de Lingüistica General. Vol. 1 (pp. 635-648). Madrid: Arco/Libros.

Crystal, D. (1972). The case of linguistics: A prognosis. British Journal of Disorders of Communication, 7, 3-16. http://doi. org/10.3109/13682827209011548

Crystal, D. (1979). Working with LARSP. London: Edward Arnold.

Crystal, D. (1981). Aspects of clinical linguistic theory and practice. Proceedings of the 1980 Annual Conference of the Australian Association of Speech and Hearing (pp. 1-25). Recuperado de http://www.davidcrystal.community.librios.com/booksand-articles/clinical-linguistics

Crystal, D. (1982). Profiling linguistic disability. London: Edward Arnold.

Crystal, D. (2001). Clinical linguistics. En M. Aronoff \& J. Ress-Miller (Eds.), The handbook of linguistics (pp. 673-682). Oxford: Blackwell.

Crystal, D. (2013). Clinical linguistics: Conversational reflections. Clinical Linguistics \& Phonetics, 27(4), 236-243. https://doi.org/ $10.3109 / 02699206.2012 .726312$ 
Crystal, D. (2019). Clinical Linguistics. En J. Damico \& M. Ball (Eds.), The Sage encyclopedia of human communication sciences and disorders (pp. 351-355). USA: Sage.

Crystal, D., Fletcher, P., \& Garman, M. (1976). The grammatical analysis of language disability. London: Edward Arnold.

Damico, J. \& Simmons-Mackie, N. (2003). Qualitative research and speech-language pathology: A tutorial for the clinical realm. American Journal of Speech-Language Pathology, 12(1), 131143. https://doi.org/10.1044/1058-0360(2003/060)

Davies, A. \& Elder, C. (2004). General introduction. Applied linguistics: Subject to discipline? En A. Davies \& C. Elder (Eds.), The handbook of applied linguistics (pp. 1-16). London: Blackwell.

Duchan, J. (2011). A history of speech-language pathology. Overview. Recuperado de http://www.acsu.buffalo.edu/ duchan/ new_history/overview.html

Fava, E. (2002). Editor's introduction. En E. Fava (Ed.), Clinical linguistics: Theory and applications in speech pathology and therapy (pp. ix-xxiii). Amsterdam: John Benjamins.

Felson, J. (2014). Case studies and their frameworks. En M. Ball, N. Müller, \& R. Nelson (Eds.), Handbook of qualitative research in communication disorders (pp. 3-16). New York: Psychology Press.

Fernández, M. (1998). El papel de la teoría y de la aplicación en la construcción de disciplinas lingüísticas. El caso de la "lingüística clínica" y áreas conexas. Revista Española de Lingüistica, 28(2), 389-419. Recuperado de http://revista.sel.edu.es/index.php/revista/article/view/1576

Fernández, M. (2002). Importancia de los modelos lingüísticos en el estudio de los trastornos comunicativos. En C. Hernández y E. Serra (Eds.), Estudios de lingüistica clínica (pp. 9-26). Valencia: Servei de Publicacions de la Universitat de València.

Fernández-Urquiza, M. (2017). Panorama actual de la lingüística clínica en España. En M. Olimpo e I. Penadés (Eds.), Investigaciones actuales en lingüistica. Vol. I. Sobre la lingüistica y sus disciplinas (pp. 195-220). Alcalá: Universidad de Alcalá.

Fernández-Uriquiza, M., Díaz, F., Moreno, V., López, M., y Simón, T. (2015). Protocolo rápido de evaluación pragmática revisado. Valencia: Universitat de València. 
Gallardo, B. (2002). Fronteras disciplinarias: pragmática y patología del lenguaje. En C. Hernández y E. Serra (Eds.), Estudios de lingüistica clínica (pp. 129-174). Valencia: Servei de Publicacions de la Universitat de València.

Gallardo, B. y Hernández, C. (2013). Lingüistica clínica. Un enfoque funcional sobre las alteraciones de lenguaje. Madrid: Arco/ Libros.

Gallardo, B. y Valles, B. (2008). Lingüística en contextos clínicos: la lingüística clínica. Lengua y Habla, 12(1), 32-50. Recuperado de http://erevistas.saber.ula.ve/index.php/lenguayhabla/ article/view/195/409

Garayzábal, E. (2004). Las alteraciones del lenguaje desde una perspectiva lingüística: el estado de la cuestión. Revista de Logopedia, Foniatría y Audiología, 24(4), 169-177. https://doi. org/10.1016/S0214-4603(04)75800-3

Goodwin, C. (2014). Foreword. En M. Ball, N. Müller, \& R. Nelson (Eds.), Handbook of qualitative research in communication disorders (pp. xi-xvi). New York: Psychology Press.

Grabe, W. (2002). Applied linguistics: An Emerging discipline for the twenty-first century. En R. Kaplan (Ed.), The Oxford handbook of applied linguistics (pp. 3-12). Oxford: Oxford University Press.

Grunwell, P. (1988). Clinical linguistics. Retrospective and prospect. En P. Grunwell (Ed.), Applied linguistics in Society (pp. 36-50). London: Warwick.

Howard, S. (2002). Clinical linguistics for students of linguistics. Centre for Languages, Linguistics and Area Studies Good Practice Guide. Recuperado de: https://www.llas.ac.uk//resources/ gpg/401

Ingram, J. (2007). Neurolinguistics. An Introduction to spoken language processing and its disorders. Cambridge: Cambridge University Press.

Jakobson, R. (1941). Kindersprache, Aphasie und allgemeine Lautgesetze. Uppsala: Universitets Arsskrift.

Jakobson, R. (1955). Aphasia as a linguistic problem. En W. Heinz (Ed.), On expressive language: Papers presented at the Clark University conference on expressive language behavior (pp. 69-81). Worcester: Clark University Press. 
Jakobson, R. (1966). Linguistic types of aphasia. En E. Carterette (Ed.), Brain function III: Speech, language, and communication (pp. 67-91). Berkeley-Los Angeles: University of California Press.

Jiménez, J. (2019). La lingüística clínica: discriminación disciplinaria y aproximación nocional desde la transversalidad. Logos: Revista de Lingüística, Filosofía y Literatura, 29(2), 286-303. https://doi.org/10.15443/rl2923

Joanette, Y. \& Ansaldo, A. (1999). Clinical note: Acquired pragmatic impairments and aphasia. Brain and Language, 68(3), 529-534. https://doi.org/10.1006/brln.1999.2126

Kemmerer, D. (2015). Cognitive neuroscience of language. New York: Psychology Press.

Lawless, S., Ries, J., \& Llorente, M. (2008). Introduction and Theoretical Foundations. En A. Llorente (Ed.), Principles of neuropsychological assessment with Hispanics (pp. 1-28). New York: Springer.

Marrero, V. (2008). El papel de la lingüística en la formación de audiólogos. En A. Moreno (Coord.), El valor de la diversidad (meta)lingüística: Actas del VIII Congreso de Lingüística General, pp. 66-77. Recuperado de http://www.lllf.uam.es/ clg8/actas/index.html

Marrero, V. y Martín, Y. (2005). Áreas de actuación del lingüista clínico:la formación y la práctica, dos perspectivas complementarias. En B. Gallardo, C. Hernández, y V. Moreno (Eds.), Lingüística clínica y neuropsicología cognitiva. Actas del Primer Congreso Nacional de lingüistica clínica Vol. 2: Lingüística y evaluación del lenguaje (pp. 201-215). Valencia: Servei de Publicacions de la Universitat de València.

Müller, N., \& Ball, M. (2013). Linguistics, phonetics, and speechlanguage pathology: Clinical linguistics and phonetics. En N. Müller \& M. Ball (Eds.), Research methods in clinical linguistics and phonetics (pp. 1-9). London: Blackwell.

Müller, N., Mok, Z., \& Keegan, L. (2014). Systemic functional linguistics and qualitative research in clinical applied linguistics. En M. Ball, N. Müller, \& R. Nelson (Eds.), Handbook of qualitative research in communication disorders (pp. 149-171). New York: Psychology Press. 
Myers, P. (2001). Toward a definition of RHD syndrome. Aphasiology, 15(10-11), 913-918. http://dx.doi. org/10.1080/02687040143000285

Paradis, M. M. (1998). The other side of language: Pragmatic competence. Journal of Neurolinguistics, 11(1-2), 1-10. https://doi. org/10.1016/S0911-6044(98)00001-3

Pavez, M. M. (1978). Algunos aspectos de la comprensión lingüística en los afásicos. RLA: Revista de Lingüística Teórica y Aplicada, $16,75-84$.

Pavez, M. M. (1980). La neurolingüística. RLA: Revista de Lingüística Teórica y Aplicada, 18, 75-100.

Pavez, M. M. (1982). Decodificación de tres tipos de estructuras sintácticas en afásicos. Thesaurus: Boletín del Instituto Caro y Cuervo, 37(2), 268-289. Recuperado de http://bibliotecadigital. caroycuervo.gov.co/610/1/TH_37_002_036_0.pdf

Pavez, M. M. (1998). Lingüística aplicada a los trastornos del lenguaje. Boletín de Filología, 37(2), 953-968. Recuperado de https://boletinfilologia.uchile.cl/index.php/BDF/article/ view/21538/22839

Perfetti, C. \& Frishkoff, G. (2008). The neural bases of text and discourse processing. En B. Stemmer \& H. Whitaker (Eds.), Handbook of the neuroscience of language (pp. 165-174). USA: Academic Press.

Perkins, M. (2011). Clinical linguistics: Its past, present and future. Clinical Linguistics \& Phonetics, 25(11-12), 922-927. https://doi. org/10.3109/02699206.2011.599471

Perkins, M. (2014). Clinical pragmatics. En J. Östman \& J. Verschueren (Eds.), Pragmatics in practice (pp. 66-92). Amsterdam: John Benjamins.

Perkins, M. \& Howard, S. (1995). Principles of clinical linguistics. En M. Perkins \& S. Howard (Eds.), Case studies in clinical linguistics (pp. 10-35). London: Whurr.

Perkins, M. \& Howard, S. (2011). Clinical linguistics. En J. Simpson (Ed.), Routledge Handbook of Applied Linguistics (pp. 111123). New York: Routledge.

Pietrosemoli, L. (1996). Coherencia y cohesión en el discurso afásico. Lengua y Habla, 1(1), 78-88. Recuperado de http:// erevistas.saber.ula.ve/index.php/lenguayhabla/article/ view/3545/3433 
Pietrosemoli, L. (2007). Análisis del discurso en poblaciones especiales: la conversación con afásicos. En A. Bolívar (Ed.), Análisis del discurso, ¿por qué y para qué? (pp. 301-320). Caracas: Los Libros del Nacional Universidad Central de Venezuela.

Pietrosemoli, L., Vera, M., González, S., y Coutín, P. (2005). Marcadores discursivos en hablantes sanos y afásicos: el caso especial de y. Boletín de Lingüística 24, 76-91. Recuperado de http://saber.ucv.ve/ojs/index.php/rev_bl/article/view/1515/1407

Powell, T. \& Ball, M. (2010). Foreword. En T. Powell \& M. Ball (Eds.), Clinical linguistics. Critical concepts in linguistics (pp. xxixxli). New York: Routledge.

Pulvermüller, F. (2002). The neuroscience of language. Cambridge: Cambridge University Press.

Quilis, A. y Hernández, C. (1990). Lingüistica española aplicada a la terapia del lenguaje. Madrid: Gredos.

Rosell, V. y Hernández, C. (Coords.) (2014). MetAphAs. Protocolo de exploración de habilidades metalingüisticas naturales en la afasia. España: Nau Llibres/Universitat de València.

Sandoval, M. (2018). Aproximaciones históricas de la disciplina fonoaudiológica en el Perú y en Latinoamérica. En J. Castro (Ed.), Introducción a la lingüística clínica (pp. 103-116). Lima: Pontificia Universidad Católica del Perú, PUCP.

Serra, E. (2013). La perspectiva funcional en Lingüística clínica. LynX. Panorámica de Estudios Lingüísticos, 12, 113-122.

Stemmer, B. (2008). Neuropragmatics: Disorders and Neural Systems. En B. Stemmer \& H. Whitaker (Eds.), Handbook of the neuroscience of language (pp. 175-188). USA: Academic Press.

Trim, J. (1963). Linguistics and speech pathology. En S. Mason (Ed.), Signs, signals and symbols: A presentation of the British approach to speech pathology and therapy (pp. 33-43). Springfield: Charles C. Thomas.

Whitaker, H. (1971). On the representation of language in the human brain. Edmonton: Linguistic Research.

Wilkinson, R. (2006). Applying conversation analysis to aphasic talk: From investigation to intervention. Revue Française de Linguistique Appliquée, 11(2), 99-110. https://doi.org/10.3917/ rfla.112.0099 